\title{
THE CAUSES AND CONSEQUENCES OF NON-NORMALITY IN PREDICTING THE PROPERTIES OF RECOMBINANT INBRED LINES
}

\author{
H. S. POONI, J. L. JINKS and M. A. CORNISH \\ Department of Genetics, University of Birmingham, Birmingham B15 2TT
}

Received 1.xi.76

\begin{abstract}
SUMmary
The contribution of two of the major causes of non-normality in the distribution of pure-breeding lines derivable by single seed descent from an $\mathrm{F}_{2}$, namely, linear epistasis and genotype $\times$ micro-environment interaction, are examined theoretically by algebraic models and computer simulations. It is shown that all the parameters required for predicting the distribution of the pure-breeding lines including the sign and magnitude of the coefficients of skewness and kurtosis which measure the deviation from normality, can be estimated from the basic set of $\mathrm{P}_{1}, \mathrm{P}_{2}, \mathrm{~F}_{1}, \mathrm{~F}_{2}, \mathrm{~B}_{1}$ and $\mathrm{B}_{2}$ generations and an $\mathrm{F}_{2}$ triple test cross.

These predictions are shown to be satisfactory in practice for nine characters in the generations and families derived from the cross between varieties 1 and 5 of Nicotiana rustica. Although the distributions of $80 \mathrm{~F}_{11}$ families derived by single seed descent from this cross display non-normality which is attributable to epistasis and genotype $\times$ environment interaction, predictions of their properties which assume normality are satisfactory providing that we make a correction to the expected means of the distributions equal to the epistatic component [i]. This correction accounts for considerably more of the discrepancies between observations and predictions than all other causes including non-normality. In practice, therefore, it appears to be the only correction that needs to be made for data of the kind we have analysed in which there are moderate levels of epistasis and medium to high heritabilities.
\end{abstract}

\section{InTRODUGTION}

IN order to make useful predictions about the properties of the recombinant pure-breeding lines that can be derived by single seed descent from the $F_{2}$ of a cross between a pair of such lines, Jinks and Pooni (1976) assumed that the line means were approximately normally distributed even where, as in the presence of directional epistasis, this was unlikely to be true. Nevertheless the predictions were satisfactory for all practical purposes. In this paper we examine two major causes of non-normality, epistasis and genotype $x$ environment interactions. Using theoretical models, simulations and practical examples we show the direction and the magnitude of the deviations from normality that they produce and the consequential effects on the usefulness of the predictions we can make in their presence.

\section{EFFEct of EPISTASIS}

Statistically, the non-normality of a distribution is jointly measured by its third and fourth moments. The moment which is associated with the asymmetry of the distribution around its mean is termed "skewness" and 
its estimate $g_{1}$ (the coefficient of skewness) for a sample size of $n$, is calculated as

$$
\left[\Sigma f_{i}\left(x_{i}-\bar{x}\right)^{3} / \Sigma f_{i}\right] /\left[\Sigma f_{i}\left(x_{i}-\bar{x}\right)^{2} / \Sigma f_{i}\right]^{\frac{3}{2}}
$$

(Snedecor and Cochran, 1967). For a population of pure-breeding lines derived by single seed descent from the $F_{2}$ of a cross between a pair of lines, the expected value of $g_{1}$ in the presence of digenic interactions is given by the expression:

$$
\left[6 \Sigma d_{j} d_{k} i_{j k}+6 \Sigma i_{j s} i_{k s} i_{j k}\right] /\left[\Sigma d_{j}^{2}+\Sigma i_{j k}^{2}\right]^{\frac{3}{2}}
$$

Here, $d_{j}$ and $d_{k}$ represent the additive effects of alleles at $j$ th and $k$ th locus and $i_{j k}, i_{j s}$ and $i_{k s}$ are respectively the additive $\times$ additive interaction effects of $j$ th and $k$ th, $j$ th and $s$ th and $k$ th and sth loci as defined on the $\mathrm{F}_{\infty}$ metric (Van der Veen, 1959).

The fourth moment, kurtosis, provides a statistical measurement of the relative peakedness (or flatness) of a distribution in terms of the projected frequencies in the central peak in relation to the density of the observations falling in its shoulders and tails. The estimate of $g_{2}$, the coefficient of kurtosis, is usually obtained from the expression

$$
\left[\left\{\Sigma f_{i}\left(x_{i}-\bar{x}\right)^{4} / \Sigma f_{i}\right\} /\left\{\Sigma f_{i}\left(x_{i}-\bar{x}\right)^{2} / \Sigma f_{i}\right\}^{2}\right]-3 ;
$$

and on our model its theoretical expectation is given by the ratio of

$$
\begin{aligned}
\Sigma d_{j}^{4} & +\sum i_{j k}^{4}+6 \Sigma d_{j}^{2} d_{k}^{2}+6 \Sigma d_{j}^{2} \sum i_{j k}^{2}+6 \Sigma i_{j k}^{2} i_{s t}^{2} \\
& +24 \Sigma d_{j} d_{k}\left(\sum i_{j s} i_{k s}+\sum i_{j s} i_{s k}+\sum i_{s j} i_{s k}+\sum i_{s j} i_{k s}\right) \\
& +24 \Sigma i_{j k} i_{j s} i_{k t} i_{s t}+24 \Sigma i_{j k} i_{k s} i_{j t} i_{s t}+24 \Sigma i_{j s} i_{j t} i_{k s} i_{s t} \\
& +24 \Sigma i_{j s} i_{j t} i_{k s} i_{k t} \text { to }\left\{\Sigma d_{j}^{2}+\sum i_{j k}^{2}\right\}^{2}, \text { minus } 3 .
\end{aligned}
$$

With the assumption that $d_{j}=d$ over all the $K$ loci and that $i_{j k}=i$ for every one of the $\frac{1}{2} K(K-1)$ pairs of loci, the expectations of $g_{1}$ and $g_{2}$ simplify to

$g_{1}=\left\{3 K(K-1) d i^{2}+K(K-1)(K-2) i^{3}\right\} /\left\{K d^{2}+\frac{1}{2} K(K-1) i^{2}\right\}^{\frac{3}{2}}$

and

$$
\begin{aligned}
g_{2}=\left[\left(\{K(1+3(K-1))\} d^{4}+\left\{\frac { 1 } { 2 } K ( K - 1 ) \left(1+3\left(\frac{1}{2} K(K-1)-1\right)+6(K-2)\right.\right.\right.\right. \\
\left.\left.(K-3))\} i^{4}+\{3 K(K-1)(5 K-8)\} d^{2} i^{2}\right) /\left\{K d^{2}+\frac{1}{2} K(K-1) i^{2}\right\}^{2}\right]-3 .
\end{aligned}
$$

In these simplified forms one can see more clearly how the magnitude and the direction of $g_{1}$ and $g_{2}$ directly reflect the magnitude and the direction of the epistatic component [i]. Nevertheless, a better appreciation of the situation can be obtained by simulation. In fig. 1 are presented three simulated distributions each for a random sample of 500 pure-breeding lines derived by single seed descent from a cross between a pair of inbreds differing at $K=10$ loci. In the first sample there is no epistasis while in the second and third samples epistasis is present at the level of $\Sigma i_{j k}^{2}=\frac{1}{10} \Sigma d_{j}^{2}$, the epistasis being of the complementary type in the second sample and of duplicate type in the third (see also the figures from Snape and Riggs, 1975). While, however, these distributions demonstrate the effect of epistasis they 
1

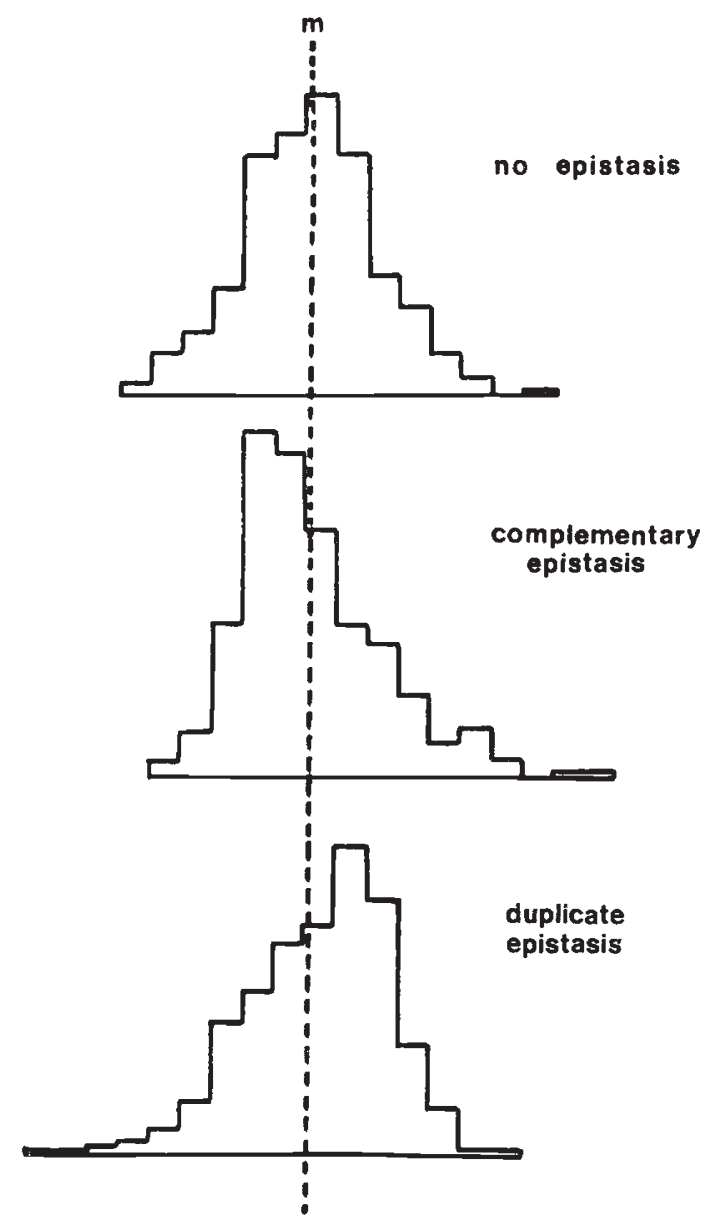

FIG. 1. Distributions of Samples of 500 Inbreds from three different simulations.

are based on a heritability of one $\left(h_{n}^{2}=1 \cdot 00\right)$ and therefore do not take into consideration the important influence of the environment and genotype $x$ environment interactions.

\section{RANDOM ENVIRONMENTAL EFFEGT AND $G \times E$ INTERAGTION}

A general effect of introducing random environmental variation on these discrete distributions of the genotypic scores is to produce continuity and to restore normality or at least to offset some of the non-normality produced by epistasis. The extent to which this occurs, however, will be dependent on the proportion of the total variation in the population attributable to random environmental causes. To illustrate this, theoretical estimates of $g_{1}$ and $g_{2}$ for various levels of epistasis and heritability $\left(h_{n}^{2}\right)$ have been tabulated in table 1. These show the expected amelioration by the random environmental variation of the deviations from normality.

The environmental variation displayed by different pure-breeding lines when exposed to micro-environmental variation, however, is not always 
TABLE 1

The expected values of $\mathrm{g}_{1}$ (coefficient of skewness) and $\mathrm{g}_{2}$ (coefficient of kurtosis) for various levels of epistasis and heritability $\left(\mathrm{h}_{n}^{2}\right)$ values. A random environmental variation is assumed when $\mathrm{h}_{n}^{2}$ is less than

$1 \cdot 0$ and skerwess is always positive for complementary epistasis and negative for duplicate epistasis

\begin{tabular}{|c|c|c|c|}
\hline \multirow{2}{*}{$\begin{array}{l}\text { Heritability } \\
\qquad \mathrm{h}_{n}^{2}\end{array}$} & \multicolumn{3}{|c|}{ Level of epistasis } \\
\hline & $0 \cdot 1$ & 0.25 & $0 \cdot 50$ \\
\hline & \multicolumn{3}{|c|}{ Skewness } \\
\hline 1.0 & $\pm 1 \cdot 17$ & \pm 1.53 & $\pm 2 \cdot 01$ \\
\hline 0.75 & \pm 0.95 & \pm 1.37 & \pm 1.71 \\
\hline $0 \cdot 50$ & \pm 0.67 & \pm 1.00 & $\pm 1 \cdot 30$ \\
\hline $0 \cdot 25$ & $\pm 0 \cdot 32$ & \pm 0.51 & \pm 0.71 \\
\hline \multirow[t]{2}{*}{$0 \cdot 10$} & \pm 0.10 & $\pm 0 \cdot 17$ & \pm 0.25 \\
\hline & \multicolumn{3}{|c|}{ Kurtosis } \\
\hline 1.0 & $1 \cdot 42$ & $2 \cdot 52$ & $4 \cdot 19$ \\
\hline 0.75 & 1.08 & $2 \cdot 50$ & 4.03 \\
\hline $0 \cdot 50$ & 0.51 & $1 \cdot 49$ & $2 \cdot 69$ \\
\hline $0 \cdot 25$ & $-0 \cdot 40$ & $0 \cdot 07$ & 0.75 \\
\hline $0 \cdot 10$ & -1.23 & -1.07 & -0.81 \\
\hline
\end{tabular}

random or homogeneous over the lines. A significant part of the environmental variation is often due to the differential response of different genotypes to the micro-environmental effects. In these circumstances the environmental variation instead of reducing the non-normality due to epistasis may itself produce non-normality. These two causes of non-normality may, therefore, reinforce or cancel out each other's contributions to non-normality depending on whether they have the same or opposing signs. The direction of the genotype $\times$ environment contribution will itself be determined by the sign of the covariance between the mean and variance of the pure-breeding lines, which has the expectation $2 \sum e_{j} d_{i} g_{i j}$, where $e_{j}$ is the additive environmental effect and $g_{i j}$ the genotype $\times$ environment interaction between $d_{i}$ and $e_{j}$. Since $d_{i}$ is always positive the sign of the covariance is essentially determined by the signs of $e_{j}$ and $g_{i j}$. If both have predominantly the same sign, whether positive or negative, the covariance and hence the skewness will be positive. If on the other hand they have predominantly opposite signs the covariance and skewness will be negative. The covariance, however, may be zero even in the presence of significant genotype $\times$ environment interactions. This will occur if the signs of $e_{j}$ and $g_{i j}$ are independent of one another. In these circumstances the interactions will produce no skewness but may still produce kurtosis. They will do so, for example, if the zero covariance results from above average variances being associated with average means and below average variances with high and low extreme means equally, or vice versa.

We can determine the sign and magnitude of this covariance from the means and variances of the pure-breeding families derived by single seed descent and test its significance as the correlation coefficient. We can also determine its sign if not its precise magnitude by deriving the covariance of mean and variance among the $\mathrm{P}_{1}, \mathrm{P}_{2}$ and $\mathrm{F}_{1}$ families (Perkins and Jinks, 1970) or amongst the families of an $\mathrm{F}_{2}$ triple test cross (Perkins and Jinks, 1971). Equally, the sign and the magnitude of sensitivity parameter 
RECOMBINANT INBRED LINES

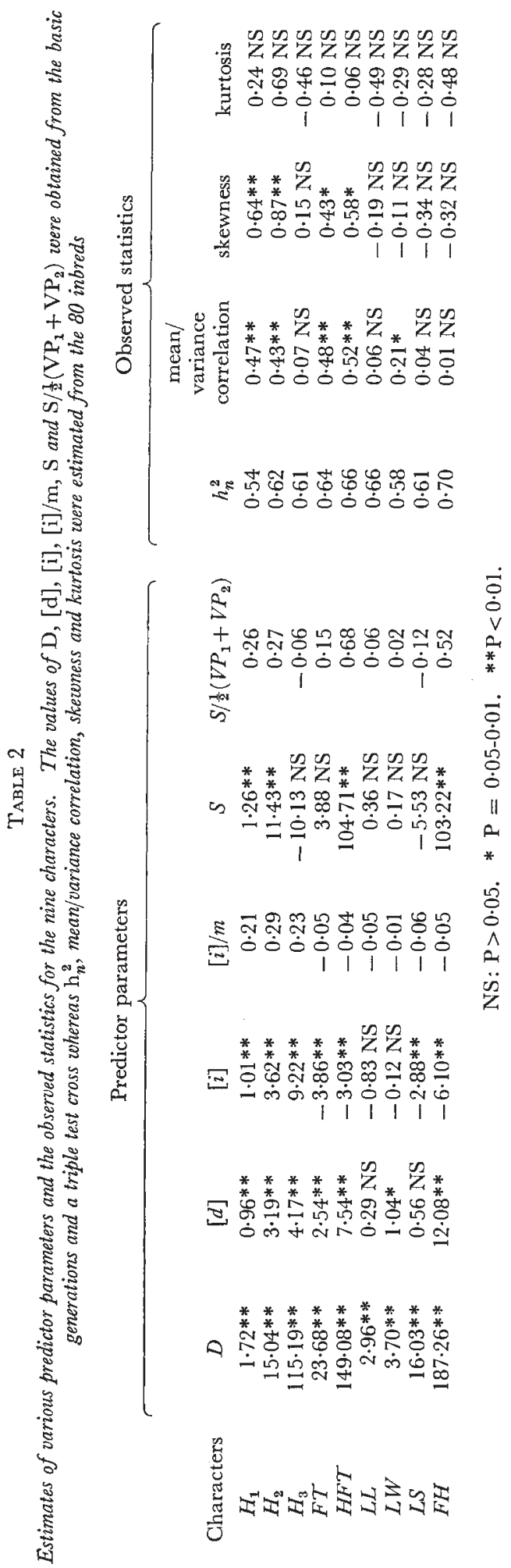


$S=\frac{1}{2}\left(V P_{1}-V P_{2}\right)=2 \Sigma e_{j} g_{i j}$ in which $V P_{1}$ is the variance of the parental line with the larger score, will establish the sign and proportionate magnitude of the genotype $x$ environment interaction covariance.

\section{EXPERIMENT AND RESULTS}

The experimental material consists of:

(i) The 80 pure-breeding $F_{11}$ families produced by single seed descent from the $F_{2}$ generation of a cross between varieties 1 and 5 of Nicotiana rustica (Pooni and Jinks, 1976).

(ii) A triple test cross set of families produced by crossing these 80 inbreds to $P_{1}, P_{2}$ and the $F_{1}$ of varieties 1 and 5 .

(iii) The basic set of six families $\left(P_{1}, P_{2}, F_{1}, F_{2}, B_{1}\right.$ and $\left.B_{2}\right)$ of the cross of varieties 1 and 5 .

All the families were raised in a single experiment. All plants were individually randomised. Each family in sets (i) and (ii) was represented by 10 plants and the six generations in set (iii) by $120,140,140,240,160$ and 200 plants, respectively. Nine metrical traits were recorded; the three developmental heights $\left(H_{1}, H_{2}\right.$ and $\left.H_{3}\right)$ taken at successive fortnightly intervals after transplanting into the field; flowering time $(F T)$ and height at flowering (HFT); leaf length $(L L)$ and leaf width $(L W)$ on the largest leaf blade; leaf spread $(L S)$ across the plant of the two leaves involving the largest leaf and the final height $(F H)$ of individual plants at the end of the season. All characters were scored in centimetres (to the nearest $0.5 \mathrm{~cm}$ ) except $F T$ which was scored in days.

TABLE 3

Dependence of Skewness and Kurtosis on epistasis $([\mathrm{i}] / \mathrm{m})$ and environmental sensitivity $\mathrm{S} / \frac{1}{2}\left(\mathrm{VP}_{\mathbf{1}}+\mathrm{VP}_{2}\right)$ as expressed in the form of regression coefficients the estimates of which are obtained from a multiple regression analysis

\begin{tabular}{|c|c|c|}
\hline \multirow[b]{2}{*}{ Independent variable } & \multicolumn{2}{|c|}{ Dependent variable } \\
\hline & Skewness & Kurtosis \\
\hline Epistasis $([i] / m)$ & $2 \cdot 11^{*} \pm 0.81$ & $1.59 \mathrm{NS} \pm 0.87$ \\
\hline $\begin{array}{l}\text { Environmental } \\
\text { sensitivity } \\
S / \frac{1}{2}\left(V P_{1}+V P_{2}\right)\end{array}$ & $0.77 \mathrm{NS} \pm 0.44$ & $0.55 \mathrm{NS} \pm 0.47$ \\
\hline
\end{tabular}

Two types of statistics can be derived from these data. The first are the estimates of the parameters which are required to predict the properties of the pure-breeding lines derivable from this cross by single seed descent. The second are the estimates of the parameters which describe the observed properties of the 80 lines that have actually been derived in this way. The first include the additive genetic component of variance $D$ which we have estimated from the triple test cross (ii) and [d], [i] and $S$ which we have estimated from the basic generations (iii). To facilitate some of the comparisons we wish to make between characters we have also standardised the estimates of [i] as [i] $/ m$ and of $S$ as $S / \frac{1}{2}\left(V P_{1}+V P_{2}\right)$. The second type of 
estimate includes the narrow heritability $h_{n}^{2}$, the correlation of family means and their variance, and the coefficients of skewness and kurtosis all of which have been obtained from the 80 pure-breeding lines themselves (i). These estimates for each of the nine characters are listed in table 2.

Reference to table 2 shows an obvious relationship over the nine characters between our standardised predictors of non-normality, [i]/m and $S / \frac{1}{2}\left(V P_{1}+V P_{2}\right)$, and our observed deviations from normality $g_{1}$ and $g_{2}$. The results of a multiple regression analysis in which the two predictors were used as the independent variables and the coefficients of skewness and kurtosis as dependent variables are summarised in table 3.

In table 4 are presented the two standard predictions of Jinks and Pooni (1976) of the proportions of pure-breeding lines which are expected to have a higher score than $\mathrm{P}_{1}$ or a lower score than $\mathrm{P}_{2}$ the first prediction assuming no [i] the second allowing for the presence of [i] but in both cases assuming normality. Also presented are the observed proportions among the 80 purebreeding lines and the discrepancies between the observed and expected proportions as absolute magnitudes.

\section{Discussion}

From the theoretical expectations in section 2 and the simulations in fig. $l$ it is quite clear that there is a direct and predictable relationship between the occurrence, magnitude and direction of skewness as measured by the coefficient $g_{1}$ and the presence, magnitude and sign of the linear additive $\times$ additive interaction component $\Sigma i_{j k}$. There is also a relationship though more complex between the coefficient of kurtosis $g_{2}$ and $\Sigma i_{j k}$. But while kurtosis in general increases with the magnitude of the epistasis there is no simple relationship between the sign of the epistasis and that of the kurtosis. These are, however, genotypic expectations and while it is with these that our predictions are concerned, in practice we shall usually be examining phenotypic distributions in which random environmental variation is superimposed upon the genetic variation.

The best way of examining the effect of random environmental variation on $g_{1}$ and $g_{2}$ is by simulation studies of the kind summarised in table 1 . These show that both skewness and kurtosis are affected by decreasing the heritability and hence increasing the contribution of random microenvironmental effects. Kurtosis, however, is more sensitive to this decrease irrespective of the level and direction of the epistasis and for moderate levels of epistasis it reaches its smallest value at intermediate values of the heritability. Skewness on the other hand, is least important when the contribution of such random environmental effects is at its greatest. In fact for heritability values below 0.5 it is unlikely that the levels of epistasis encountered in practice would lead to significant skewness.

The coefficient of skewness, $g_{1}$, is either significant $(P<0.05)$ or highly significant $(\mathrm{P}<0.01)$ for four of the nine characters (table 2). Furthermore, its direction and magnitude follows closely that of our standardised predictor [i] $/ m$ with a positive and significant regression of $2 \cdot 11 \pm 0 \cdot 81$ (table 3 ). Thus the theoretical relationship holds good in practice.

In contrast the estimates of the coefficient of kurtosis, $g_{2}$, are nonsignificant in all the cases (table 2) even though their magnitudes and signs vary between +0.69 and -0.49 as would be expected from the simulations 


\section{TABLE 4}

The observed and expected proportion of inbreds falling outside the parental range for various characters. The expected proportions are worked out by assuming no epistasis ([d] only) and by allowing for the epistatic component ([d] and [i]). $P_{1}$ is the larger scoring parent (V1 or V5) for the character concerned

Proportion of inbreds falling outside parental range

\begin{tabular}{|c|c|c|c|c|c|c|c|c|}
\hline \multirow[t]{3}{*}{ Character } & \multicolumn{2}{|c|}{$\begin{array}{l}\text { Observed } \\
\text { proportions }\end{array}$} & \multicolumn{4}{|c|}{ Expected proportions } & \multicolumn{2}{|c|}{$\begin{array}{c}\text { †Summed discrepancies } \\
\text { between observed and } \\
\text { expected }\end{array}$} \\
\hline & \multirow[b]{2}{*}{$>\bar{P}_{1}$} & \multirow[b]{2}{*}{$<\overline{\mathrm{P}}_{2}$} & \multicolumn{2}{|c|}{ from $[d]$ alone } & \multicolumn{2}{|c|}{ from $[d]$ and $[i]$} & \multirow[b]{2}{*}{ with $[d]$} & \multirow[b]{2}{*}{ with $[d]$ and $[i]$} \\
\hline & & & $>\overline{\mathrm{P}}_{1}$ & $<\bar{P}_{2}$ & $>\overline{\mathrm{P}}_{1}$ & $<\overline{\mathrm{P}}_{2}$ & & \\
\hline$H_{1}$ & $0 \cdot 10$ & $0 \cdot 50$ & $0 \cdot 23$ & $0 \cdot 23$ & 0.07 & 0.52 & 0.40 & 0.05 \\
\hline $\mathrm{H}_{2}$ & 0.08 & $0 \cdot 64$ & $0 \cdot 21$ & $0 \cdot 21$ & 0.04 & 0.54 & $0 \cdot 56$ & $0 \cdot 14$ \\
\hline$H_{3}$ & $0 \cdot 18$ & $0 \cdot 70$ & $0 \cdot 35$ & 0.35 & $0 \cdot 11$ & $0 \cdot 68$ & $0 \cdot 52$ & 0.09 \\
\hline$F T$ & $0 \cdot 58$ & 0.06 & $0 \cdot 30$ & $0 \cdot 30$ & $0 \cdot 61$ & $0 \cdot 10$ & $0 \cdot 52$ & 0.07 \\
\hline$H F T$ & 0.30 & 0.20 & $0 \cdot 27$ & 0.27 & $0 \cdot 36$ & $0 \cdot 19$ & $0 \cdot 10$ & 0.07 \\
\hline$L L$ & $0 \cdot 60$ & 0.31 & 0.43 & 0.43 & $0 \cdot 63$ & $0 \cdot 26$ & $0 \cdot 29$ & 0.08 \\
\hline$L W$ & 0.40 & $0 \cdot 30$ & 0.29 & $0 \cdot 29$ & 0.32 & 0.27 & $0 \cdot 12$ & $0 \cdot 11$ \\
\hline$L S$ & $0 \cdot 68$ & $0 \cdot 26$ & 0.44 & $0 \cdot 44$ & 0.72 & $0 \cdot 19$ & $0 \cdot 42$ & $0 \cdot 11$ \\
\hline$F H$ & 0.40 & 0.13 & $0 \cdot 19$ & 0.19 & 0.33 & 0.09 & 0.27 & 0.11 \\
\hline
\end{tabular}

$\uparrow$ Discrepancies between observed and expected proportions are worked out for each character by summation of the absolute deviations between the observed and expected values.

for characters with medium $h_{n}^{2}$ values and a moderate level of epistasis (tables 1 and 2). The sample size of 80 pure-breeding lines, however, is much smaller than the 500 that would be required to obtain significance for an epistatic level of 0.1 and a $h_{n}^{2}$ value of 0.6 (Cornish 1976).

The largest value of $g_{2}$ is obtained for the character $H_{2}$ which has a highly significant value for [i] and the largest value for [i] $/ m$ (table 2) and over all the characters there is a positive regression of $g_{2}$ on $[\mathrm{i}] / \mathrm{m}$ of $1.59 \pm 0.87$ (table 3). This regression, however, is not significant which is not surprising in that none of the $g_{2}$ values is significant and we do not expect a simple linear relationship between them (table 1 ).

While the estimates of $g_{1}$ and $g_{2}$ show the expected positive relationship with our standardised sensitivity parameter (table 3 ) neither of the regression coefficients is significant. Again this is not surprising in that only four of the estimates of the sensitivity parameter and none of the $g_{2}$ values are significant. That the theoretical relationship holds, however, is shown conclusively by replacing the sensitivity parameter in the multiple regression analysis by the observed correlation between family means and the variances among the 80 pure-breeding lines, whereupon both regression coefficients become significant $\left(1 \cdot 63^{* *} \pm 0 \cdot 16\right.$ and $\left.1 \cdot 27^{*} \pm 0 \cdot 38\right)$. There can be no doubt, therefore, that in practice directional interactions between the genotypes and the micro-environmental variation produce both skewness and kurtosis and our failure to establish a significant causal relationship using our standardised sensitivity predictor must be due to the unreliability of the estimates of the predictor (see section 6).

Although we know that both epistasis and genotype $\times$ environment interaction have produced significant non-normality for more than half of the characters studied we have nonetheless proceeded with the prediction of the 
proportion of pure-breeding lines that are expected to fall outside of the parental range assuming normality (table 4) in order to assess the consequences of the failure of this assumption. As reported in our previous paper (Jinks and Pooni, 1976) the discrepancies between observed and expected proportions are much larger when no allowance is made for [i] than when allowance is made for its presence. Nevertheless, even after allowing for [i] there are still residual discrepancies between the observed and expected. For example, for the first character in table $4\left(H_{1}\right)$ the summed discrepancies when no allowance is made for [i] are 0.40 and after allowing for it are 0.05 . Hence allowing for [i] reduces the discrepancies by 0.35 and all other causes of discrepancies including non-normality account for the remaining 0.05 . The corresponding average values over all nine characters are 0.24 and 0.09 respectively. Thus even though some of these characters do not have a significant value for [i] nonetheless the simple linear correction for [i] on average accounts for more of the discrepancies between observed and expected proportions than all other causes combined. These other causes include non-normality and the sampling errors of our predictions and of the observed statistics. In addition we are predicting the proportion of genotypes that are expected to fall outside of the parental range and observing the proportion of phenotypes. The latter is a real distinction in these data where the heritability varies between 0.54 and 0.70 (table 2). We can, of course, readily accommodate these deviations from a heritability of one in our prediction equations (Jinks and Pooni, 1976), although it is the proportion of genotypes we wish to predict in most circumstances. The only clear indication that non-normality may be a contributor to the residual discrepancies is provided by character $H_{2}$ (table 4) which has the largest skewness (table 2) and the largest discrepancy. The importance of the other causes, however, is equally shown by the characters $H_{3}, L L, L W, L S$ and $F H$ which also have above average residual discrepancies but show no significant skewness.

\section{Conclusions}

These studies have demonstrated that directional epistasis and genotype $\times$ environment interactions are two major causes of non-normality in the distribution of pure-breeding lines. This non-normality may be anticipated and allowed for in making predictions by observing the significance and direction of $\Sigma i_{j k}$ and the correlation between family means and variances. Estimates of [i] obtained from the basic generations, as in the present paper, provide satisfactory predictions although they could be misleading if there were a high degree of dispersion of the interacting alleles in the parental lines. A better prediction of the correlation between family means and variances than the one used in the present paper is obtainable from a covariance analysis of $\left(\tilde{L}_{1 i}+L_{2 i}\right)$ and $\left(\sigma_{L 1 i}^{2}+\sigma_{L 2 i}^{2}\right)$ values from an $\mathrm{F}_{2}$ triple test cross (Perkins and Jinks, 1971). Since this design is also the best source of the other predictors its use would not involve any additional experimentation.

A further step in the present studies would be to use our predictors of non-normality to generate appropriate non-normal distributions, the probabilities of which could then be integrated to predict the proportion of pure-breeding lines falling outside of the parental range by having a higher score than $\mathrm{P}_{1}$ or a lower score than $\mathrm{P}_{2}$. While this, however, may be worthy 
of further investigation there appears to be little justification for attempting this except for perhaps character $\mathrm{H}_{2}$ in the present study.

Particularly impressive is the confirmation of our earlier finding (Jinks and Pooni, 1976) that by using [i] to make a linear correction to the expected mean of the distribution of pure-breeding lines we can remove most of the discrepancies between the predicted and observed results. And while there are residual discrepancies not even in the worst case (character $\mathrm{H}_{2}$ in table 4) are these discrepancies more than of borderline significance $(P=0 \cdot 05-0 \cdot 02)$. They are therefore very unlikely in practice to lead to an incorrect decision either in choosing between alternative crosses or in determining the sample size required to yield a predictable proportion of pure-breeding lines whose performance exceeds some predetermined score with some predetermined probability of success.

Acknowledgments.-We are indebted to Dr M. J. Kearsey who developed the computer package upon which the simulations are based and for his advice on their further development.

\section{REFERENCES}

CORNISH, M. A. 1976. A study of the causes of non-normality in the distribution of inbred lines derived by single seed descent. M.Sc. thesis, Dept. of Genetics, University of Birmingham.

JINKs, J. L., AND POONI, H. s. 1976. Predicting the properties of recombinant inbred lines derived by single seed descent. Heredity, 36, 253-266.

PERKINS, J. M., AND JINKS, J. L. 1970. Detection and estimation of genotype-environmental, linkage and epistatic components of variation for a metrical trait. Heredity, 25, 157-177.

PERKINS, J. M., AND JINKS, J. L. 1971. Analysis of genotype $\times$ environment interaction in triple test cross data. Heredity, 26, 203-209.

POONI, H. s., AND JiNks, J. L. 1976. The efficiency and optimal size of triple test cross designs for detecting epistatic variation. Heredity, 36, 215-227.

SNAPE, J. W., AND RIGGs, T. J. 1975. Genetical consequences of single seed descent in the breeding of self pollinating crops. Heredity, 35, $211-219$.

sNedecor, G. W., AND COCHRAN, w. G. 1967. Statistical Methods. Iowa State University Press, Ames, Iowa, U.S.A.

VAN DER VEEN, J. H. 1959. Tests of non-allelic interaction and linkage for quantitative characters in generations derived from two diploid pure lines. Genetica, $X X X, 201-232$. 